\title{
Catalan Sign Language ellipsis, role shift, and the QUD
}

\author{
David Blunier (University of Geneva) \\ david.blunier@unige.ch \\ Giorgia Zorzi (UPF, Barcelona; HVL, Bergen) \\ giorgia.zorzi88@gmail.com
}

\begin{abstract}
This paper is concerned with the interpretation of context-dependent elements such as $I X_{1}, I X_{2}$ and $I X_{(H E R E)}$ in Catalan Sign Language (LSC) under role shift and ellipsis. We show that their behavior in both environments sheds light on ellipsis mechanisms, which share essential properties with anaphora and discourse reference, and speech reports constructions.
\end{abstract}

Keywords: Pronouns, ellipsis, role shift, Question Under Discussion, Catalan Sign Language.

\section{Introduction}

Since the pioneering work of David Kaplan (Kaplan 1989), it is often assumed that elements such as $I$, you, here and now should be given a special status within semantic theories, most notably because of their context-sensitive properties: $I$, for instance, always refers directly to the speaker of the actual utterance it is used in, and is famously insensitive to intensional operators such as attitude verbs like say.

(1) Sam said to Maria that I am in love with you.

In (1), I refers to the actual speaker who is reporting what Sam said, and you refers to the actual addressee of what has been reported. This property, among others, sets elements such as $I$ and you apart from similar ones such as third person he or she, which do not possess this rigidity in reference. Sign languages, however, tend to behave differently in allowing anaphoric reference of first and second person pronouns in attitude reports. In this paper, we explore the behavior of such 'role-shifted' indexicals in interaction with ellipsis constructions, such as VP-ellipsis, which reveal puzzling restrictions on pronominal reference. The paper is structured as follows: Section 1 introduces the main phenomenon this study is concerned with, namely, the interaction of role shift with ellipsis in sign languages. Section 2 introduces the methodology used for elicitation, as well as the relevant LSC data, to which we provide a formal analysis in Section 3; Section 4 concludes. 

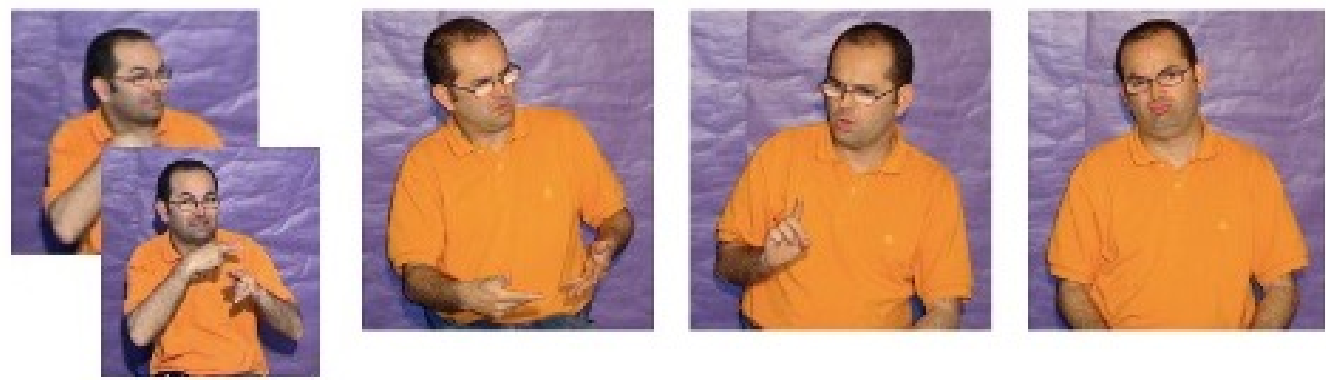

Figure 1: Non-manual markers of role shift: eyegaze and body shift, head tilt, facial expressions (Quer 2013: 13).

\subsection{Indexicals and role shift in sign languages}

In sign languages, pronominal elements share a common structure in that they all consist of a combination of a pointing gesture, an index, and a locus, which serves to identify a given referent in the signing space (Sandler and Lillo-Martin 2006, among others). In the case of first and second person, the loci associated with the index are the body of the signer and the position of the actual addressee, respectively. However, in most sign languages (if not all, see Herrmann and Steinbach 2012), first and second person pronouns can be used anaphorically in intensional constructions through role shift. Role shift is a complex construction allowing for the signer to report utterances or thoughts from a distinct, but first-personal, perspective, in a quotational manner. ${ }^{1}$

When using role shift, a signer will use non-manual markers (NMMs) such as body lean, head tilt, and eyegaze contact break with the addressee. These NMMs, illustrated in Figure 1 , typically take scope over the reported sentence.

As mentioned above, under role shift, indexical pronouns $I X_{1}$ and $I X_{2}$ are 'shifted away' from the actual speaker and addressee to refer to the agent and addressee of the reported utterance or thought, as in (2) from Catalan Sign Language (LSC): ${ }^{2}$

$$
\begin{aligned}
& \mathrm{RS}_{a} \\
& \text { 'Manel }{ }_{a} \text { thinks that he } a \text { won't give me }{ }_{b} \text { anything at all.' }
\end{aligned}
$$

(LSC, Quer 2011: 280)

In (2), the signer is reporting a sentence that Manel told him: the first person pronoun $I X_{1}$ under role shift refers to Manel, the agent of the reported utterance, and not to the actual signer; the shifted second person agreement reference under role shift, instead, refers to the actual signer. Under role shift, the first person pronoun loses its indexical value to be interpreted as a third person anaphoric pronoun.

1. Whether or not sign language role shift could be reduced to a form of quotation as commonly used in spoken languages is still much debated. See most notably Davidson (2015) and Maier (2018) for arguments in favor of a quotational analysis, and Schlenker (2017a, 2017b) for an alternative view.

2. The glosses conventions used in this paper follow the ones developed in the SIGN-HUB project grammar. The number indexes 1, 2 and 3 refer respectively to first, second and third person singular. The letter indexes are used to identify the referent the $I X$ pronoun is coindexed with; they do not necessarily refer to a specific location in the signing space. 


\subsection{The interaction of indexicals with ellipsis}

Similar to most spoken languages, pronouns in sign languages give rise to the so-called 'strict/sloppy readings' under ellipsis, as in (3), an example from Italian Sign Language (LIS): ${ }^{3}$

(3) GIANNI $a$ SECRETARY POSS $a$ VALUE. PIERO SAME.

a. 'Gianni $a$ values his $a$ secretary, Piero ${ }_{b}\langle$ values his $a$ secretary, too.' (strict reading)

b. 'Gianni $a$ values his $a$ secretary, Piero ${ }_{b}\left\langle\right.$ values his $_{b}$ secretary $\rangle$, too.' (sloppy reading)

(LIS, Cecchetto et al. 2015: 229)

As initially observed by, among others, Dahl (1973) and Williams (1977), VP-ellipsis sites tend to generate ambiguities whenever antecedents contain a referential pronoun: as a consequence, its elided counterpart can either refer to the topmost DP Gianni, giving rise to a strict reading (3a), or to the closer 'remnant' DP, Piero, licensing a sloppy reading (3b).

\subsection{A restriction on indexicals in Italian Sign Language}

As illustrated in (3), sign languages also display the strict/sloppy alternation. What is more, as demonstrated by Cecchetto et al. (2015) for LIS, a restriction seems to arise in configurations involving an indexical pronoun under role shift: when the antecedent contains a roleshifted indexical, it cannot be interpreted 'strictly' in the ellipsis site, contrary to its third person counterpart. This restriction is illustrated in (4) and (5).

(4) GIANNI $a$ SAY IX $3 a$ MARIA KISS. PIERO SAME.

strict $\boldsymbol{}$, sloppy $\checkmark$

'Gianni ${ }_{a}$ said that he kissed Maria. Piero $_{b}\langle$ said that he $a / b$ kissed Maria〉, too.'

GIANNI $_{a}$ SAY $\frac{\mathrm{RS}_{a}}{\mathrm{IX}_{1} a \text { MARIA KISS. PIERO SAME. }}$

strict $\boldsymbol{X}$, sloppy $\checkmark$

'Gianni ${ }_{a}$ said that he ${ }_{a}$ kissed Maria. Piero ${ }_{b}\left\langle\right.$ said that he $*_{a / b}$ kissed Maria〉, too.'

(LIS, adapted from Cecchetto et al. 2015: 229)

In order to explain this restriction, Cecchetto et al. (2015) adopt the context-shifting analysis for indexicals under role shift of Schlenker (2017a). Under this view, the NMMs used to license role shift are analyzed as introducing a dedicated operator in the logical form of reported sentences, whose role is to shift the coordinates of the actual context against which the embedded indexical is evaluated. As a result, the indexical comes out being interpreted not referring to the speaker, but to the subject of the matrix clause, Gianni:

$$
\llbracket \mathrm{SAY}-O P_{i} \phi \rrbracket^{g, c}=\lambda x^{\prime} \cdot \lambda w^{\prime} \llbracket \phi \rrbracket^{g\left[i \rightarrow x^{\prime}\right], w^{\prime}}
$$

The shifting operator $S A Y-O P$, when fed a proposition $\phi$, changes the speaker and world coordinates of the actual context $c$ on the interpretation function in order to deliver a function from individuals and worlds to truth values, a centered proposition in the sense of Lewis (1979). The first person indexical is thus bound (alongside the world of evaluation of the reported sentence) and allowed to refer back to the subject of the matrix verb SAY, i.e., Gianni.

The absence of a strict reading in sentences like (5) leads Cecchetto et al. (2015) to argue that the ellipsis site contains a copy of $S A Y-O P$, thus vindicating an approach to ellipsis that assumes an 'identity in form' between the antecedent and the ellipsis clause, in the spirit of analyses such as those of Sag (1976), Fiengo and May (1994), Merchant (2001), Rudin (2019).

3. Here and throughout the paper, we indicate elided material between 〈angled brackets〉. 
The reasoning goes as follows: since the silent indexical in (5) can only be interpreted as referring to the external argument of the elided verb $S A Y$, i.e., Piero, the lack of the other reading follows from the presence of an elided operator binding the indexical in the elided clause, henceforth blocking a strict interpretation.

\section{Catalan Sign Language data}

\subsection{Methodology}

The LSC data presented in this work were collected from two Deaf native consultants with a particular linguistic awareness of LSC and accustomed to data elicitation. Data collection sessions took place both live and online using the same methodology. First, the consultants were presented with a context under three different modalities: signed in LSC, pictured and written in glosses. Both the written context and glosses were in Spanish, the written language they are most comfortable with. After seeing the context, the consultants were presented with the sentence that we were expecting to have different readings depending on the context. Three different contexts were showed for the same sentence (matrix strict, matrix sloppy, and embedded), but each interpretation was discussed pairing one context at the time with the sentence. Discussion about the interpretation was conducted in LSC. Each sentence was recorded and then discussed again in the subsequent sessions to make sure the judgments were consistent. ${ }^{4}$

\subsection{Strict/sloppy interpretations of $\mathrm{IX}_{1}$ under role shift}

In LSC, no difference between role-shifted first person reports and indirect third person ones was observed. Interestingly, both sentences license strict and sloppy interpretations, regardless of presence or absence of role shift, in contrast to LIS. The two readings, though, matrix strict and matrix sloppy, strictly depend on the context previously introduced. Consider the following examples of the same target sentences, in which each context favors one interpretation over the other.

(7) Context: The secretary says: "I will give a present to Josep". Giorgia says: "The secretary will give a present to Josep". ${ }^{5}$
a. SECRETARY $a$ SAY IX $3 a$ JOSEP $_{b}{ }_{a}$ PRESENT-GIVE $_{3} b$ IX $_{3 c}$ GIORGIA $_{c}$ TOO. $\mathrm{RS}_{a}$
b. SECRETARY $a$ SAY $\overline{\text { IX }_{1 a} \text { JOSEP }_{b} 1 a \text { PRESENT-GIVE }_{3} b}$, IX $_{3 c}$ GIORGIA $_{c}$ TOO.
(video)
'The secretary $a$ said that she'll give a present to Josep, Giorgia ${ }_{c}\left\langle\right.$ said that she $_{a /{ }^{*} c}$ will give a present to Josep $\rangle$, too.'
$\checkmark$ strict (secretary) $\boldsymbol{X}$ sloppy (Giorgia)

(8) Context: The secretary says: "I will give a present to Josep". Giorgia says: "I will give a present to Josep".

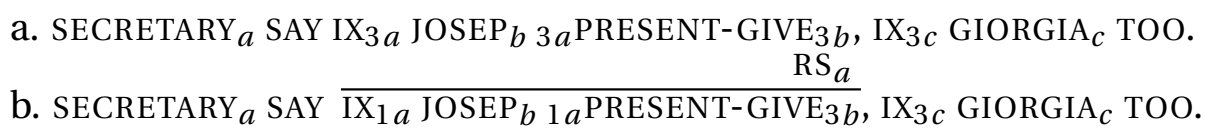

(video)

(video)

4. Online sessions were carried out with the support of a PowerPoint presentation and sentences were recorded using screen recording.

5. Informants were provided with contexts both signed and written in glosses as the following, corresponding to the sloppy reading: SECRETARY: "IX ${ }_{1}$ JOSEP PRESENT-GIVE"

GIORGIA: "IX 1 JOSEP PRESENT-GIVE" 
'The secretary $a$ said that she'll give a present to Josep, Giorgia ${ }_{c}\langle$ said that she $* a / c$ will give a present to Josep $\rangle$, too.'

$\boldsymbol{X}$ strict (secretary) $\boldsymbol{V}$ sloppy (Giorgia)

When sentences were presented out of the blue, a "narrow scope" interpretation of the ellipsis site was favored by the informants, in which the ellipsis site corresponds to the embedded sentence only (embedded reading), excluding the matrix verb $S A Y$, as in (9).

(9) SECRETARY $a$ SAY $\frac{\mathrm{RS}_{a}}{\mathrm{IX}_{1 a} \mathrm{JOSEP}_{b}{ }_{1} a \text { PRESENT-GIVE }_{3} b}$, IX $_{3 c}$ GIORGIA $_{c}$ TOO.

'The secretary $a$ said that she'll give a present to Josep and Giorgia ${ }_{c}$ 〈will give a present to Josep〉, too.'

Observations for both LIS and LSC are summarized in Table 1.

Table 1: Readings of the ellipsis clause with and without role shift in LIS and LSC.

\begin{tabular}{|c|c|c|}
\hline & No RS & $\mathrm{RS}$ \\
\hline LIS & $\begin{array}{l}\text { Matrix sloppy: } \checkmark \\
\text { Matrix strict: } \checkmark \\
\text { Embedded:? }\end{array}$ & $\begin{array}{l}\text { Matrix sloppy: } \checkmark \\
\text { Matrix strict: } \boldsymbol{X} \\
\text { Embedded: ? }\end{array}$ \\
\hline LSC & $\begin{array}{l}\text { Matrix sloppy: } \checkmark \\
\text { Matrix strict: } \checkmark \\
\text { Embedded: } \checkmark\end{array}$ & $\begin{array}{l}\text { Matrix sloppy: } \checkmark \\
\text { Matrix strict: } \checkmark \\
\text { Embedded: } \checkmark\end{array}$ \\
\hline
\end{tabular}

These observations on the LSC data lead to two main questions: i) how can we explain the different readings available for the ellipsis site in LSC?; ii) how can we justify similar interpretations of third person and shifted first person pronouns under ellipsis in LSC?

\section{Analysis: ellipsis sites as answers to QUDs}

In order to capture the LSC data above, we propose to analyze ellipsis as a form of discourse reference - in the sense of Wasow (1972), Hardt (1993), Kehler (2000), Poppels (2020), among others - whose content is partially determined by the Question Under Discussion.

\subsection{Ellipsis as discourse reference}

In Question Under Discussion (QUD) models, ellipsis is subsumed under general anaphora mechanisms allowing discourse reference, and is not subject to specific licensing conditions like parallelism or identity. Like other pronominal forms, ellipsis reference is sensitive to general discourse constraints like precedence and salience, as well as the "aboutness" of the current discourse it appears in.

This is where the QUD approach is directly relevant for our concerns. In a QUD model such as the one of Roberts (1996/2012), discourse can be viewed as a hierarchical set of question/answer pairs aimed at sharing statements about the way things are (Stalnaker 1978). Participants in a conversation aim at answering these questions following a defined strategy of inquiry that relies on prosodic, semantic and pragmatic cues. Following Keshet (2013), Elliott, Nicolae, and Sudo (2016) and Kehler (2016), we straightforwardly apply this model to ellipsis by positing that the content of elided constituents can be resolved against (sometimes implicit) QUDs in the discourse structure. As an example, consider (10): 
(10) Every boy in John's class hoped Mrs. Smith would pass him. In John's ${ }_{j}$ case, I think she WILL $\left\langle\right.$ pass $\left.\operatorname{him}_{j}\right\rangle$.

(Kehler 2016: 10)

The elided VP of the second sentence admits a sloppy reading of the form I think she will pass John that is unexpected in this configuration, since the pronoun him in the antecedent is bound by the quantifier phrase Every boy. This is a major problem for identity theories of ellipsis which posit a "parallelism" requirement between the elided VP and its antecedent, be it syntactic, semantic, or both (Fox 2000; Merchant 2001). As a solution, Kehler (2016) proposes to assume that the alternatives against which ellipsis is computed are not directly provided by the antecedent clause, but rather by the PP As for John, which introduces a contrastive topic within the discourse structure. Contrastive topics are used to introduce new QUDs (Büring 2003) which, in turn, provide the relevant set of alternatives against which the ellipsis site can be resolved. Thus, in the above example, the ellipsis site is not computed directly against the antecedent Every boy in John's class hoped Mrs. Smith would pass him but rather, against the implicit question What about John ${ }_{j}$ ? Do you think she will pass him h $_{j}$ ?

Such a model treats ellipsis phenomena on a par with other forms of discourse anaphora, such as pronominal reference: contrary to identity approaches, in which antecedents provide the syntactic/semantic material licensing elliptical structure, the QUD model vindicates a referential approach in which antecedents are just a convenient way to promote discourse referents that can otherwise be inferred, as in (10).

\subsection{Predicting matrix and embedded readings}

The above model can straightforwardly derive the first set of data regarding the scope of ellipsis. Recall that, depending on the provided context, sentences like the ones in $(7,8$, 9) can trigger a "matrix" interpretation, incorporating the matrix verb and the ambiguous pronoun $(7,8)$, or an "embedded" interpretation, in which the ellipsis site only consists of the embedded sentence and does not give rise to the strict-sloppy alternation (9). This is in line with observations initially made by Urmson (1952) about the status of embedding verbs such as say or think, which can trigger a so-called parenthetical reading (Simons 2007, 2019) as in the following example:

(11) A: Why didn't Louise come to the meeting yesterday?

B: I heard that she's out of town.

(Simons 2007: 2)

In (11), B's utterance felicitously answers A's question because only the embedded sentence she's out of town is deemed relevant, while the matrix verb is treated as a parenthetical. Put differently, the matrix verb is considered being not at-issue and, therefore, is not interpreted as introducing a QUD corresponding to How do you know that Louise is out of town? that B's utterance should aim at answering. The ongoing QUD is thus identified on pragmatic grounds by identification of which VP (matrix or embedded) serves as the main point of utterance (Simons 2007, 2019).

The same reasoning can be applied to our LSC data: in $(7,8)$, the matrix VP is identified as the main point of utterance, in a context where the saying event is made salient. As a consequence, two QUDs can be established, giving rise to the strict/sloppy alternation.

The QUD that correctly derives the sloppy interpretation for the ellipsis site in (8) corresponds to the set of propositions of the form $x$ said that $x$ gave a present to Josep, like in (12). 
(12) a. QUD (8): Who ${ }^{x}$ said that $x$ gave a present to Josep?

(Sloppy reading)

b. $\lambda p . \exists x(p=\lambda w . x$ said that $x$ gave a present to Josep in $w)$.

As for the strict interpretation, it is obtained through identification of another QUD, corresponding to the one in (13).

(13) a. QUD (7): Who said that the secretary gave a present to Josep?

(Strict reading)

b. $\lambda p . \exists x(p=\lambda w . x$ said that the secretary gave a present to Josep in $w)$.

The QUD in (13) corresponds to the set of propositions of the form $x$ said that the secretary gave a present to Josep, identifying the elided pronoun with the referent the secretary and enforcing a strict interpretation. In a similar fashion, the embedded reading is obtained when the matrix verb is disregarded as a possible candidate for introducing a relevant QUD: this happens, for instance, in contexts where it is unclear whereas two distinct utterances were made, as in (9). In that case, only the content of the embedded clause is interpreted as being at issue, introducing a QUD of the form in (14).

(14) a. QUD (9): Who gave a present to Josep?

(Embedded reading)

b. $\lambda p . \exists x(p=\lambda w . x$ gave a present to Josep in $w)$.

As a consequence, the only possible value that the ellipsis site can have is a proposition of the form $x$ gave a present to Josep, with no associated ambiguities.

\subsection{Predicting strict/sloppy alternations with indexicals}

What remains to be explained is the lack of interpretative differences between plain, nonshifted utterances such as $(7 \mathrm{a}, 8 \mathrm{a})$, and role-shifted ones like $(7 \mathrm{~b}, 8 \mathrm{~b})$, which fail to exhibit the pattern observed for LIS by Cecchetto et al. (2015). In LSC, the presence vs. absence of role shift does not seem to generate semantic differences, at least not at the propositional level. ${ }^{6}$ Recall that, in their analysis, Cecchetto et al. (2015) interpret the unavailability of strict readings in role shifted reports to be an argument for the presence of articulated, unpronounced material within the ellipsis site, as advocated by e.g. Chung (2006) and Merchant (2013). This is so because, according to their analysis, the context-shifting operator SAY-OP defined in (6) is directly copied from the antecedent in the ellipsis clause, forcing all the indexicals within its scope to be shifted to the closest matrix subject (in the cited example (5), Piero).

This line of analysis might be appealing, but cannot be straightforwardly applied to our LSC data, which does not seem to behave like LIS in this respect. Crucially, we suggest that, on the contrary, the LSC data vindicate a different stance towards ellipsis, according to which elided sites are driven by discourse reference mechanisms analogous to those at play for pronoun and anaphora resolution, as suggested by Poppels and Kehler (2017), Poppels (2020) and Miller and Hemforth (2020). According to this line of analysis, ellipsis sites essentially behave as unspecified pro-forms that are anaphoric to discourse referents. As mentioned in the previous section, the antecedent retrieving mechanisms are sensitive to a variety of factors, including contextual information, salience, and the QUD (see, among others, Poppels (2020) and Miller and Hemforth (2020) for experimental data). Following this line of reasoning, we propose that the strict/sloppy interpretation of third person and shifted first person

6. Role-shifted utterances seem to exhibit presuppositional restrictions that their non-shifted counterparts do not, such as a verbatim requirement which presupposes that the form of the original utterance was identical to the one of the report (Schlenker 2017b). 
pronouns in the ellipsis site is allowed by phi-features being ignored, a phenomenon that is attested in a broad range of ellipsis phenomena.

Consider first an example of VP ellipsis in American Sign Language, from Schlenker (2014):

(15) IX $\mathrm{IX}_{3 p l} 6$ FRENCH SWIMMER LIKE PEOPLE SUPPORT IX ${ }_{3 p l}$. IX IX $_{b}$ GERMAN SWIMMER SAME $a, b$. 'The six French swimmers $a$ like people who support them ${ }_{a}$. The German swimmer ${ }_{b}$ does 〈like people who support $\left.\operatorname{him}_{b}\right\rangle$, too.' $\quad$ (ASL, adapted from Schlenker 2014: 26)

Note that in (15), the plural features on the pronoun $I X_{3 p l}$ are uninterpreted in the ellipsis site. The same holds for other ellipsis phenomena, such as gapping:

(16) MARINA $_{a}$ JORDI $_{b}$ WATCH $_{3}$ GIVE $_{3} b$, MARC $_{c}$ JORDINA $_{d}$ PLANT.

'Marina gave Jordi a watch and Marc <gave> Jordina a plant.'

(LSC, Zorzi 2018: 341)

In (16) the person features associated with the agreeing verb GIVE - referring to the loci attributed to referents MARINA and JORDINA, respectively - are left uninterpreted in the ellipsis site. Similarly, in our canonical examples (7b, 8b), the first-person feature (analyzed here as shifted towards the reported speaker, SECRETARY) does not restrict the range of possible individuals that could be referred to by the ellipsis site.

The approach presented so far focuses on the explanation of the LSC data, but do not address the restrictions on the readings observed in role-shifted sentences like (5) in LIS, i.e., the exclusive availability of the sloppy reading under RS. Since the pragmatics of discourse reference are not assumed to be language-dependent, adopting the model presented for LSC would predict that the restrictions observed in LIS amount either to contextual ones, or should be taken as default preferences for ellipsis resolution in LIS, something that should be addressed in further research. ${ }^{7}$

\subsection{Another indexical: $\boldsymbol{X}_{(H E R E)}$}

In line with previous research on shifted indexicals in LSC, we also tested the behavior of the locative indexical $I X_{(H E R E)}$ under ellipsis. In his pioneering study, Quer (2005) observed that, in a single role-shifted report, two indexicals could refer to different contexts, even though both were under the scope of role shift markers. This is illustrated in (17): ${ }^{8}$

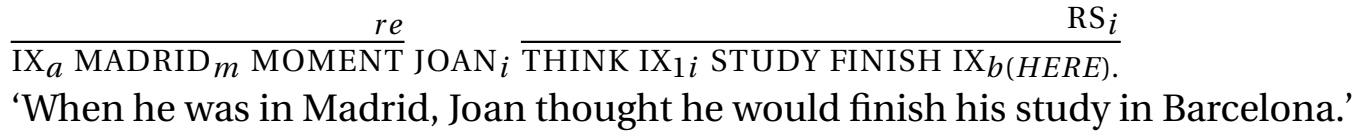

(LSC, adapted from Quer 2005: 154)

In the example above, the first person indexical $I X_{1}$ is shifted towards $J O A N$, the reported speaker, while the locative indexical $I X_{(H E R E)}$ denotes the actual place of utterance, Barcelona. This was taken by Quer (2005) as a counterexample to the shift together constraint proposed by Anand and Nevins (2004) for indexical shift in spoken languages.

Our data, however, suggest that things might be more intricate. In fact, reference of the indexical $I X_{(H E R E)}$ seems to be ambiguous in absence of further contextual information:

7. We thank Carlo Geraci for bringing this point to our attention.

8. The notation "re" refer to "raised eyebrows". 
(18) Context: Gemma meets Brendan in San Sebastian. Brendan says: "I like to live here". Later, Gemma reports to Josep:

BRENDAN $_{a}$ SAY $_{\mathrm{IX}_{1 a} \text { LIVE IX }_{b / s s(H E R E)} \mathrm{LIKE}_{a}}$

(video)

'Brendan ${ }_{a}$ said that he ${ }_{a}$ likes to live here ${ }_{b / s s}$.'

$I X_{(H E R E)}=$ Barcelona $/$ San Sebastian

With no further specification, the indexical $I X_{(H E R E)}$ in the role-shifted report in (18) can assume two different values: the place of the actual utterance, Barcelona, or that of the report, San Sebastian. The ambiguity can be drastically reduced upon indicating a specific location in the matrix clause preceding the report, as in (19):

(19) Context: same as (18).

$\mathrm{IX}_{s s}$ SAN SEBASTIAN BRENDAN $_{a}$ SAY $_{\mathrm{IX}_{1 a} \text { LIVE IX }_{s s(H E R E)} \mathrm{LIKE}}$

(video)

'While in San Sebastian, Brendan ${ }_{a}$ said that he likes to live here $_{s s}$. '

In the above example, the preferred interpretation of $I X_{(H E R E)}$ is San Sebastian, thanks to both mentions of the reported location and the context, in contrast to Quer's (2005) results (cp. his (23)).

Even more interestingly, the behavior of $I X_{(H E R E)}$ under ellipsis closely mirrors the one presented in $(7 \mathrm{~b}, 8 \mathrm{~b})$ for the first person indexical $I X_{1}$. In the following example, the context allows for a sloppy reading of $I X_{(H E R E)}$ in the ellipsis site:

(20) Context: Gemma meets Brendan in San Sebastian. Brendan tells her: "I like to live here". She then goes on to visit her friend Javier in Madrid, who tells her: "I like to live here". Back in Barcelona, Gemma tells Josep about what her friends told her:

BRENDAN $_{a}$ SAY $_{\mathrm{IX}_{1} a}$ LIVE IX $_{b / s s(H E R E)} \mathrm{LIKE}_{a}$ JAVIER TOO.

(video)

'Brendan ${ }_{a}$ said that he likes to live here $_{b / s s}$, Javier $_{j}\left\langle\right.$ said that he $_{j}$ likes to live here $\left.{ }_{b / s s / m}\right\rangle$ too.'

$\left\langle I_{(H E R E)}\right\rangle=$ Barcelona/San Sebastian/Madrid

In (20), the elided indexical $I X_{(H E R E)}$ can assume all three possible values provided by the context: Barcelona (actual place of utterance), San Sebastian (Brendan's location) and Madrid (Javier's location).

As its first-person counterpart $I X_{1}$, the elided value of $I X_{(H E R E)}$ is sensitive to contextual information. In the following example, the second saying event is modified, changing the available QUDs and, in turn, constraining the possible values for the ellipsis site, making it possible only for the strict reading to arise:

(21) Context: Gemma meets Brendan in San Sebastian. Brendan tells her: "I like to live here". She then goes on to visit her friend Javier in Madrid, who tells her: "Brendan likes to live here". Back in Barcelona, Gemma tells Joseph about what her friends told her:

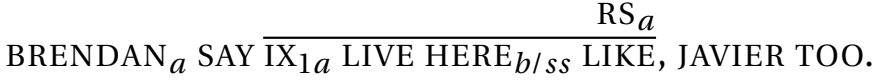

(video)

'Brendan ${ }_{a}$ said that he $a$ likes to live $\operatorname{IX}_{b / s s(H E R E)}$, Javier ${ }_{j}$ 〈said that he ${ }_{a}$ likes to live here $\left._{b / s s / * m}\right\rangle$ too.'

$\left\langle I X_{(H E R E)}\right\rangle=$ Barcelona/San Sebastian $/{ }^{*}$ Madrid

Note that the values of the two elided indexicals $I X_{1}$ and $I X_{(H E R E)}$ do not vary independently: in (21), the value of $I X_{1}$ has to refer to Brendan, since $I X_{(H E R E)}$ refers to Brendan's location, reminding the shift together effect as defined by Anand and Nevins (2004) and Anand (2006) for indexical shift in spoken languages. Again, ambiguity is maintained through possible identification of different QUDs, corresponding to different readings. Whenever two saying events are explicitly introduced in the context, the matrix reading is made available, making it possible for the strict/sloppy reading of indexical $I X_{(H E R E)}$ to arise. 
(22) a. QUD (20): Where does $x$ like to live?

(sloppy reading)

b. $\lambda p . \exists x . \exists y . y$ is the location of $x(p=\lambda w . x$ likes to live in $y$ in $w)$.

(23) a. QUD (21): Where does Brendan like to live?

(strict reading)

b. $\lambda p . \exists x . x$ is Brendan's location ( $p=\lambda w$. Brendan likes to live in $x$ in $w$ ).

Note also that, contrary to example $(7 \mathrm{~b}, 8 \mathrm{~b})$, the indexical/unshifted reading of $I X_{(H E R E)}$ is always available, both inside or outside ellipsis - contrary to its first person counterpart. This is not an isolated feature of LSC, similar results having been reported for DGS as well (see Herrmann and Steinbach 2012).

How should this asymmetry be accounted for? As a tentative answer, we would like to suggest that this is a consequence of the pragmatics of role shift. Following Zucchi (2004), we analyze the NMMs of role shift mentioned above as introducing a presupposition that the variable introduced by the pronoun be identified with the author of the report. If no such individual cannot be identified, the result is presupposition failure, and communication fails. But - and this is crucial - no such failure can arise, since such a speaker is always grammatically provided qua subject of the matrix clause. This explains why $I X_{1}$ must shift under role shift: if the speaker were to refer to himself using $I X_{1}$, he would not use role shift in the first place, unless the speaker is self-quoting, or quoting their past self. However, this is not the case for $I X_{(H E R E)}$, whose adverbial function allows it to remain unspecified. When this is the case, contextual information is crucial to retrieve indexical reference intended by the speaker: this explains the data in (19), where SAN SEBASTIAN is provided in the matrix clause, allowing $I X_{(H E R E)}$ to refer back to it under role shift.

Another argument in favor of such an analysis is provided by the second person indexical $I X_{2}$ under role shift: when no addressee is specified in the report, $I X_{2}$ can refer to the addressee of the actual utterance or the shifted one. However, if the addressee of the reported context is introduced as a discourse referent, $I X_{2}$ must have a shifted reading. This is illustrated in (24): ${ }^{9}$

(24) Context: The secretary tells to Gemma: "I work better than you do". Later that day, Gemma reports what she has been told to Xavi:

a. SECRETARY $a_{a}$ SAY IX $_{1 a}$ WORK $\frac{/ \text { mejor } /}{1_{a} \mathrm{ARC}_{2} / x}$.

(video)

'The secretary ${ }_{a}$ said that she ${ }_{a}$ works better than you $_{g, x}$.' Second pers $=$ Gemma $/$ Xavi
$\mathrm{RS}_{a}$
b. SECRETARY $a$ SAY GEMMAg IX $_{1 a}$ WORK $\frac{/ \text { mejor/ }}{1_{a} \mathrm{ARC}_{2} /{ }^{*} x}$.
(video)
'The secretary ${ }_{a}$ said that she ${ }_{a}$ works better than you $_{g,{ }^{*} x}$.' Second pers= Gemma

In this example, the second person indexical feature is fully disambiguated when provided with a lexically specified referent, as in (24b); when not, as in (24a), both addressees can be selected under role shift, with a clear preference for the shifted interpretation if the NMMs used for the report involve the body of the signer shifting away from the actual addressee (Xavi in the examples above).

9. The notation "/mejor/" represents the mouthing of the word "mejor" in Spanish, which means 'better'. 


\section{Conclusion}

The LSC data presented in this paper provide new insights on the interpretation of indexicals $I X_{1}, I X_{2}$ and $I X_{(H E R E)}$ under role shift and ellipsis, supporting a model where ellipsis is a form of entity-retrieving mechanism in discourse, similar to discourse anaphora in general. The matrix and embedded readings can be resolved against QUDs in the discourse structure; the strict/sloppy interpretation of third person and shifted first person pronouns in the ellipsis site, instead, can be allowed by phi-feature being ignored in the ellipsis site. This approach is radically different from the one put forth in Cecchetto et al. (2015) where the shifting of indexicals under role shift in LIS is due to a context-shift operator à la Schlenker (2017a). If the ellipsis site does not feature any covert operator of that type, nothing guarantees that the pronominal referent intended in the ellipsis site will refer to the closest attitude holder (i.e., the subject of the matrix verb SAY introducing RS for the interpretation of the shifted $I X_{1}$ ). Rather, ellipsis under the matrix reading is fully ambiguous, and such ambiguity can only be lifted by pragmatics, through identification from the hearer of the relevant QUD addressed by the speaker.

\section{References}

Anand, Pranav. 2006. “De De Se.” PhD diss., MIT.

Anand, Pranav, and Andrew Nevins. 2004. "Shifty operators in changing contexts." In Semantics and Linguistic Theory, 14:20-37.

Büring, Daniel. 2003. “On D-trees, beans, and B-accents.” Linguistics and philosophy 26 (5): 511-545.

Cecchetto, Carlo, Alessandra Checchetto, Carlo Geraci, Mirko Santoro, and Sandro Zucchi. 2015. "The syntax of predicate ellipsis in Italian Sign Language (LIS).” Lingua 166:214235.

Chung, Sandra. 2006. "Sluicing and the lexicon: The point of no return." In Proceedings of the annual meeting of the Berkeley Linguistics Society, 31:73-91.

Dahl, Östen. 1973. “On so-called "sloppy identity"." Synthese, 81-112.

Davidson, Kathryn. 2015. "Quotation, demonstration, and iconicity." Linguistics and Philosophy 38 (6): 477-520.

Elliott, Patrick D, Andreea Nicolae, and Yasutada Sudo. 2016. "The sticky reading: VP ellipsis without parallel binding." In Semantics and Linguistic Theory, 24:640-655.

Fiengo, Robert, and Robert May. 1994. Indices and identity. MIT press.

Fox, Danny. 2000. Economy and semantic interpretation. MIT press.

Hardt, Daniel. 1993. "Verb phrase ellipsis: Form, meaning, and processing." PhD diss., University of Pennsylvania.

Herrmann, Annika, and Markus Steinbach. 2012. "Quotation in sign languages." In Quotatives: Cross-linguistic and Cross-disciplinary Perspectives, edited by Annika Herrmann and Marku Steinbach, 203-228. Amsterdam: John Benjamins. 
Kaplan, David. 1989. “Demonstratives.” In Themes From Kaplan, edited by Joseph Almog, John Perry, and Howard Wettstein, 481-563. Oxford University Press.

Kehler, Andrew. 2000. "Coherence and the resolution of ellipsis." Linguistics and Philosophy 23 (6): 533-575.

- 2016. "On QUD-based licensing of strict and sloppy ambiguities.” In Semantics and Linguistic Theory, 25:512-532.

Keshet, Ezra. 2013. “Sloppy identity unbound.” In Semantics and Linguistic Theory, 23:412431.

Lewis, David. 1979. “Attitudes de dicto and de se.” The Philosophical Review 88 (4): 513-543.

Maier, Emar. 2018. "Quotation, demonstration, and attraction in sign language role shift." Theoretical Linguistics 44 (3-4): 265-276.

Merchant, Jason. 2001. The syntax of silence: Sluicing, islands, and the theory of ellipsis. Oxford University Press on Demand.

—. 2013. "Voice and ellipsis." Linguistic Inquiry 44 (1): 77-108.

Miller, Philip, and Barbara Hemforth. 2020. "Acceptability of VP ellipsis: discourse conditions vs. syntactic identity." In CUNY conference on Human Language Processing. Amherst, MA, USA.

Poppels, Till. 2020. “Towards a Referential Theory of Ellipsis.” PhD diss., University of California, San Diego.

Poppels, Till, and Andrew Kehler. 2017. "Overcoming the identity crisis: Novel evidence for a referential theory of verb phrase ellipsis." In Proceedings from the Annual Meeting of the Chicago Linguistic Society, 53:291-305. 1. Chicago Linguistic Society.

Quer, Josep. 2005. “Context shift and indexical variables in sign languages.” In Semantics and linguistic theory, 15:152-168.

_. 2011. "Reporting and quoting in signed discourse.” In Understanding Quotation, edited by Elke Brendel, Jörg Meibauer, and Markus Steinbach, 277-302. Berlin: Mouton de Gruyter.

- 2013. "Attitude ascriptions in sign languages and role shift." In Proceedings of the 13th Meeting of the Texas Linguistics Society, 12-28.

Roberts, Craige. 1996/2012. "Information structure: Towards an integrated formal theory of pragmatics.” Semantics and Pragmatics 5:1-69.

Rudin, Deniz. 2019. "Head-based syntactic identity in sluicing." Linguistic Inquiry 50 (2): 253-283.

Sag, Ivan A. 1976. "Deletion and logical form.” PhD diss., MIT.

Sandler, Wendy, and Diane Lillo-Martin. 2006. Sign language and linguistic universals. Cambridge: Cambridge University Press.

Schlenker, Philippe. 2014. "Iconic features.” Natural Language Semantics 22 (4): 299-356.

2017a. "Super Monsters I: Attitude and action role shift in sign language.” Semantics and Pragmatics 10. 
Schlenker, Philippe. 2017b. "Super Monsters II: Role shift, iconicity and quotation in sign language." Semantics and Pragmatics 10.

Simons, Mandy. 2007. "Observations on embedding verbs, evidentiality, and presupposition.” Lingua 117 (6): 1034-1056.

- 2019. "The Status of Main Point Complement Clauses." In Proceedings of the 23rd Workshop on the Semantics and Pragmatics of Dialogue - Full Papers. London, United Kingdom: SEMDIAL, September.

Stalnaker, Robert. 1978. “Assertion.” In Syntax and Semantics, edited by Peter Cole, 9:315332. New York: New York Academic Press.

Urmson, James O. 1952. "Parenthetical verbs.” Mind 61 (244): 480-496.

Wasow, Thomas. 1972. “Anaphoric relations in English.” PhD diss., MIT.

Williams, Edwin S. 1977. “Discourse and Logical Form.” Linguistic Inquiry 8 (1): 101-139.

Zorzi, Giorgia. 2018. “Coordination and gapping in Catalan Sign Language (LSC).” PhD diss., Universitat Pompeu Fabra.

Zucchi, Alessandro. 2004. "Monsters in the visual mode." Ms., Università degli Studi di Milano. 